\title{
Seiberg-Witten-Like Equations on 8-Manifolds without Self-Duality
}

\author{
Serhan Eker* \\ Department of Mathematics, Faculty of Science and Letters, Ağrı İbrahim Çeçen University, Ağrı, Turkey \\ *srhaneker@gmail.com
}

Received: 29 July 2018

Accepted: 12 October 2018

DOI: $10.18466 /$ cbayarfbe. 448934

\begin{abstract}
In this paper, Seiberg-Witten-like equations without self-duality are defined on 8 -dimensional manifolds. Then, non-trivial and flat solutions are given to them on $\mathbb{R}^{8}$. Finally, on 8 -real-dimensional Kähler manifolds a global solution to these equation is obtained for a given negative and constant scalar curvature.

Keywords: Seiberg-Witten equations; Spin and $\operatorname{Spin}^{c}$ geometry; Curvature; Without self-duality.
\end{abstract}

\section{Introduction}

On the 4-manifolds, Seiberg-Witten equations introduced by E. Witten are consisted of Dirac equation and Curvature equation [9]. These equations provide information about the topology and geometry of the 4 -manifolds $[3,4,6,8,9]$. To define these equations, one needs two entities as an $i \mathbb{R}$ valued connection 1 -form and a spinor field. Dirac equation can be defined on any manifold endowed with $\operatorname{Spin}^{c}$-structure. But, defining the curvature equation needs self-duality concept of two form. Since self-duality concept is meaningful only in 4 -dimension, generalized self-duality concept is given to define the curvature equation on a noun four- dimensional manifold. Accordingly, Seiberg-Witten equations are investigated up to 4 -dimensional manifolds by defining generalized self-duality concept [1,5]. On 8 -manifolds, Seiberg-Witten-like equations have been studied in $[1,2,5]$ depending on the Spin and Spin $^{c}$-structure. In [1], the author defined SeibergWitten-like equations on the Spin manifold with respect to the generalized self-duality concept and gives them local solutions. Then, in [2] these equations are constructed on the $\operatorname{Spin}^{c}$ manifolds and non-trivial local solutions are given to them. Finally, the global solutions of these equations are given on the 8-manifolds endowed with $S U(4)$-structure in [5].

The purpose of this paper is planning to give in two part. One of them is to write down Seiberg-Witten-like equations without using the self-duality concept on the $4-$ manifolds and to show similarities with the classical Seiberg-Witten equations. The other one is to define these equations on the 8 -manifolds without using the self-duality concept and to obtain a non-trivial flat solution on the 8-dimensional Riemannian manifolds. Also, to give them a global solution on the 8 -real-dimensional Kähler manifold for a given negative and constant scalar curvature.

\section{Materials and Methods}

2.1 $\operatorname{Spin}^{c}$-structure and Dirac operator

Suppose that $M$ is an orientable Riemannian manifold. Hence, there exist an open covering $\left\{U_{\alpha}\right\}_{\alpha \in \mathcal{A}}$ of $M$ with the transitions functions $g_{\alpha \beta}: U_{\alpha} \cap U_{\beta} \rightarrow S O(n)$ for $T M$. If there exists another collection of transition functions $\tilde{g}_{\alpha \beta}: U_{\alpha} \cap U_{\beta} \rightarrow \operatorname{Spin}^{c}(n)$ such that the following diagram commutes

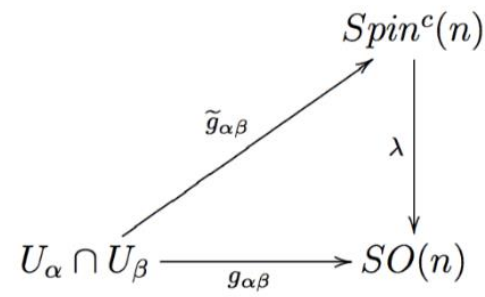

That is, $\lambda \circ \tilde{g}_{\alpha \beta}=g_{\alpha \beta}$ and the cocycle condition $\tilde{g}_{\alpha \beta}(x) \circ \tilde{g}_{\beta \gamma}(x)=\tilde{g}_{\alpha \gamma}(x)$ on $U_{\alpha} \cap U_{\beta} \cap U_{\gamma} \neq \emptyset$ is satisfied, then $M$ is called Spin $^{c}$ manifold.

On a $\operatorname{Spin}^{c}$ manifold, one can construct $P_{S O(n)}$,

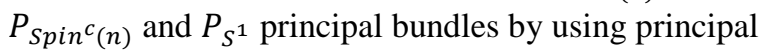
bundle construction lemma [7]. Also, by using $P_{S^{1}}$ principal bundle one can construct determinant line bundle

where

$$
\mathcal{L}:=P_{\operatorname{Spin}^{c}(n)} \times{ }_{l} \mathbb{C}=P_{S^{1}} \times_{U(1)} \mathbb{C}
$$

$$
l_{\alpha \beta}=l: U_{\alpha} \cap U_{\beta} \rightarrow \operatorname{Spin}^{c}(n) .
$$

Moreover, an associated complex vector bundle $\mathbb{S}=P_{\operatorname{Spin}^{c}(n)} \times_{\kappa_{n}} \Delta_{n}$ can be constructed by considering spinor representations

$$
\kappa_{n}: \operatorname{Spin}^{c}(n) \rightarrow \operatorname{Aut}\left(\Delta_{n}\right)
$$

where $\Delta_{n}=\mathbb{C}^{2^{\frac{n}{2}}}$. If the dimension of $M$ is even, then $\mathbb{S}$ spinor bundle splits into two pieces $\mathbb{S}=\mathbb{S}^{+} \oplus \mathbb{S}^{-}[4]$. The sections of the complex vector bundle are called spinor fields. On the complex vector bundle $\mathbb{S}$ one can define Hermitian inner product as follows: 


$$
\begin{array}{rll}
<,>: \Gamma(\mathbb{S}) \times \Gamma(\mathbb{S}) & \rightarrow & \mathbb{C} \\
([p, \Psi],[p, \Phi]) & \mapsto & <\Psi, \Phi>=\Psi \cdot \Phi .
\end{array}
$$

By using Hermitian inner product defined in (2.1) one can associate each spinor $\Psi$ to an endomorphism of $\mathbb{S}$ by the formula

$$
\begin{aligned}
\Psi \Psi^{*}: \mathbb{S} & \longrightarrow \mathbb{S} \\
\tau & \mapsto<\Psi, \tau>\Psi .
\end{aligned}
$$

Following bundle homomorphisms are useful while studying on spinors. Extended map of $\kappa_{n}$ is defined by

$$
\kappa: T M \longrightarrow \operatorname{End}(\mathbb{S}) .
$$

Some authors called the map $\kappa$ a $\operatorname{Spin}^{c}$-structure on the manifold $M$ [8].

The Clifford multiplication with $X$ is defined

$$
X \cdot \Psi:=\kappa(X)(\Psi)
$$

where $X \in \Gamma(T M)$ and $\Psi \in \Gamma(\mathbb{S})$.

A spinor covariant derivative operator $\nabla^{A}$ is obtained by using an $A: T P_{S^{1}} \rightarrow i \mathbb{R}, i \mathbb{R}$-valued 1 -form in the principal bundle $P_{S^{1}}$ and Levi-Civita connection $\nabla$ on $M$ as follows

$$
\nabla_{X}^{A} \Psi=\mathrm{d} \Psi(\mathrm{X})+\frac{1}{2} \sum_{i<j} \omega_{i j}(X) e_{i} \cdot e_{j}(\Psi)+\frac{1}{2} A(X)(\Psi)
$$

where $\Psi \in \Gamma(\mathbb{S})$ and $X \in \Gamma(T M)$.

Now we can define the Dirac operator locally as follows.

Definition 1: Let $e=\left\{e_{1}, e_{2}, \ldots, e_{n}\right\}$ be any local orthonormal frame on $U \subset M$. Then the local expression of the Dirac operatör $D_{A}=\Gamma(\mathbb{S}) \rightarrow \Gamma(\mathbb{S})$ is

$$
D_{A} \Psi=\sum_{\mathrm{i}=1}^{n} e_{i} \cdot \nabla_{e_{i}}^{A} \Psi
$$

where $\Psi \in \Gamma(\mathbb{S})$ and $A \in \Omega^{1}(M, i \mathbb{R})$. Dirac operator decomposes into $D_{A}=D_{A}^{+} \oplus D_{A}^{-}$in the case of dimension of $M$ is even.

By using $\kappa$, another bundle map $\rho$ associated each 2 -form to an endomorphism of $\mathbb{S}$, can be defined on the orthonormal frame $\left\{e_{1}, e_{2}, \ldots, e_{n}\right\}$ as follows

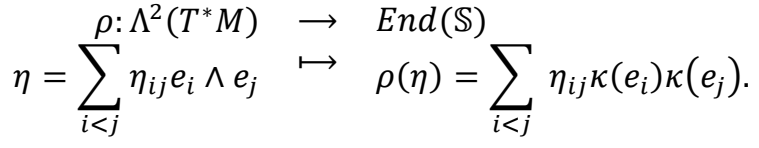

Also $\rho$ can be extend to a complex valued 2 -forms [8] such that

$$
\rho: \Lambda^{2}\left(T^{*} M\right) \otimes \mathbb{C} \rightarrow \operatorname{End}(\mathbb{S}) .
$$

Also $\rho$ can be defined on the half spinor bundles $\mathbb{S}^{ \pm}$. The half-spinor bundles $\mathbb{S}^{ \pm}$are invariant under $\rho(\eta)$ for all $\eta \in \Lambda^{2}\left(T^{*} M\right)$. That is,

$$
\begin{aligned}
& \rho(\eta)(\Psi) \in \mathbb{S}^{+}, \forall \Psi \in \mathbb{S}^{+} \\
& \rho(\eta)(\Psi) \in \mathbb{S}^{-}, \forall \Psi \in \mathbb{S}^{-} .
\end{aligned}
$$

Then, we obtain the following maps by restriction $\rho^{+}(\eta)=\left.\rho(\eta)\right|_{S^{+}}, \rho^{-}(\eta)=\left.\rho(\eta)\right|_{S^{-}}$. In this case is expressed as follows:

$$
\rho^{+}: \Lambda^{2}\left(T^{*} M\right) \otimes \mathbb{C} \rightarrow \operatorname{End}\left(\mathbb{S}^{+}\right)
$$

$$
\rho^{+}(\eta)=\rho^{+}\left(\sum_{i<j} \eta_{i j} e_{i} \wedge e_{j}\right)=\sum_{i<j} \eta_{i j} \kappa\left(e_{i}\right) \kappa\left(e_{j}\right) .
$$

Note that, the space of $i \mathbb{R}$-valued 2 -forms $\Lambda^{2}(\mathrm{M}, \mathrm{i} \mathbb{R})$ is a sub bundle of $\Lambda^{2}(\mathrm{M}, \mathrm{i} \mathbb{R}) \times \mathbb{C}$. We consider the sub bunde $W=\rho^{+}\left(\Lambda^{2}(\mathrm{M}, \mathrm{i} \mathbb{R})\right)$ of $\operatorname{End}(\mathbb{S})$ to define curvature equation.

In order to be able to give a global solution for the Seiberg-Witten-like equation defined without self-duality, the manifold must be endowed with $S U(4)$ - structure. That guarantees the existence of a Hermitian metric compatible with the complex structure on a Hermitian manifold. On the Hermitian manifold one can construct canonical $\operatorname{Spin}^{c}$ - structure and by using this structure spinorial bundle can be defined with a spinorial connection. Also, Dirac operator is associated with such a connection. As a result Seiberg-Witten-like equation without self-duality can be defined on such manifold and a global solution can be given to it.

In the following, before the global solution is given, a short brief of the Kähler manifolds is given.

\subsection{Kähler Manifolds}

On the 8-manifolds endowed with $S U(4)$-structure, there exists an almost complex structure satisfying $J: T M \longrightarrow T M, J^{2}=-I_{d}$.

A smooth manifold endowed with an almost complex structure is called an almost complex manifold and denoted by $(M, J)$.

The almost complex structure $J$ acts on the space of 1 -forms as follows:

$$
\begin{aligned}
J: T M & \rightarrow \mathrm{TM} \\
\omega & \mapsto J(\omega)(X):=\omega(J X)
\end{aligned}
$$

where $\omega \in \Gamma\left(T^{*} M\right)$ and $X \in \Gamma(T M)$. Moreover, $J$ acts on the complexification of the cotangent bundle of $\mathrm{M}$ as

$$
\begin{aligned}
J: T^{*} M \otimes_{\mathbb{R}} \mathbb{C} & \rightarrow J: T^{*} M \otimes_{\mathbb{R}} \mathbb{C} \\
\omega \otimes Z & \mapsto J(\omega) \otimes Z .
\end{aligned}
$$

Since $J^{2}=-I_{d}, \pm i$ are eigenvalues of $\mathrm{J}$. Then $T^{*} M \otimes_{\mathbb{R}} \mathbb{C}$ is the direct sum of

$$
T^{*} M \otimes_{\mathbb{R}} \mathbb{C}=\wedge^{1,0}(M) \oplus \wedge^{0,1}(M)
$$

where

$$
\begin{aligned}
& \Lambda^{1,0}(M)=\left\{Z \in T^{*} M \bigotimes_{\mathbb{R}} \mathbb{C} \mid J Z=i Z\right\} \\
& \Lambda^{0,1}(M)=\left\{Z \in T^{*} M \bigotimes_{\mathbb{R}} \mathbb{C} \mid J Z=-i Z\right\} .
\end{aligned}
$$

The space of $r$-forms is given as:

where

$$
\Lambda^{r}(M)=\sum_{a+b=r} \Lambda^{a, b}(M)
$$

$\Lambda^{a, b}(M)=\operatorname{span}\left\{x \wedge y \mid x \in \Lambda^{a}\left(\Lambda^{1,0}(M)\right), y \in \Lambda^{b}\left(\Lambda^{0,1}(M)\right)\right\}$ is the space of $(a, b)$ type complex forms. Finally, Kähler manifold is defined as follows.

Definition 2: Let $(M, J)$ be an almost complex manifold. Then, a Riemannian metric $g$ is called Hermitian metric if it is compatible with the almost complex structure $J$ :

where $X, Y \in \Gamma(T M)$.

$$
g(J X, J Y)=g(X, Y)
$$


The associated smooth 2 -form $\Phi$ defined by

$$
\Phi(X, Y)=g(X, J Y)
$$

is called the Kähler 2 -form and satisfies $\Phi(J X, J Y)=$ $\Phi(X, Y)$. If $\Phi$ is closed then M is called Kähler Manifold and the metric on $\mathrm{M}$ is called a Kähler metric.

\subsection{Dirac operator on the Kähler Manifolds}

In this section, we talk about the canonical Spin $^{c}$ - structure of a Kähler manifold and its spinor bundle with associated connection. Since the structure group of any Kähler manifold of dimension $n$ is $U(n)$, it admits a canonical $\operatorname{Spin}^{c}$ - structure given by:

$$
P_{\operatorname{Spin}^{c}(n)}=P_{U(n)} \times_{F} \operatorname{Spin}^{c}(2 n)
$$

where $F: U(n) \rightarrow \operatorname{Spin}^{c}(2 n)$ is the lifting map [4]. The associated canonical spinor bundle then has the form:

$$
\mathbb{S} \cong \Omega^{(0, *)}(M)
$$

where $\Omega^{(0, *)}(M)$ is the direct sum of $\Omega^{(0,1)}(M) \oplus \Omega^{(0,2)}(M) \oplus \ldots \oplus \Omega^{(0, i)}(M), \quad i \in \mathbb{N}$. There are two ways to include a spinorial Levi-Civita connection on $\mathbb{S}$.

The first is obtained by the extension of the connection to forms and the latter is obtained via $\operatorname{Spin}^{c}-$ structure. In this work, we mainly focused on the canonical $\operatorname{Spin}^{c}$ - structure with the following isomorphism:

$$
\mathbb{S} \cong \Omega^{(0, *)}(M) \text {. }
$$

On this bundle, we described Dirac operator defined on $\mathbb{S}$ and we give the relation with the Dirac-type operator defined on $\Omega^{(0, *)}(M)$.

In the case of Kähler manifold endowed with a canonical $\operatorname{Spin}^{c}$-structure, there is a spinorial connection $\nabla^{A}$ on the associated spinor bundle $\mathbb{S}$ induced by an unitary connection 1 -form $A$ on the determinant line bundle $\mathcal{L}$ together with the spinorial Levi-Civita connection $\nabla$. Also, on the associated spinor bundle one can describe Dirac operator as follows:

Let $\left\{e_{i}\right\} i=1, \ldots, n$ be a local orthonormal frame on $\mathrm{M}$. Then the Dirac operator $D^{A}$ is given by:

$$
D_{A} \Psi=\sum_{\mathrm{I}=1}^{n} e_{i} \cdot \nabla_{e_{i}}^{A} \Psi .
$$

Moreover, by considering Kähler manifolds with $\Omega^{(0, *)}(M)$ associated spinor bundle the Dirac type operator is defined as follows:

Let

$\bar{\partial}: \Omega^{0, r}(M) \rightarrow \Omega^{0, r+1}(M), \bar{\partial}^{*}: \Omega^{0, r}(M) \rightarrow \Omega^{0, r-1}(M)$, given by:

$\overline{\partial_{0}}=\sum_{i=1}^{n} \bar{Z}_{l}^{*} \wedge \nabla_{\bar{Z}_{l}},{\overline{\partial_{2}}}^{*}=-\sum_{i=1}^{n} \iota\left(\bar{Z}_{l}\right)^{*} \wedge \nabla_{\bar{Z}_{l}} \quad$ respectively, where $\nabla$ is the extension of the Levi-Civita connection to $\Omega^{(0, *)}(M)$ and $\iota$ is the contraction operator.

Since $\mathbb{S} \cong \Omega^{(0, *)}(M)$, one has

$$
D_{A_{0}}=\sqrt{2}\left({\overline{\partial_{0}}}+{\overline{\partial_{2}}}^{*}\right)
$$

where $A_{0}$ is the Levi-Civita connection of the line bundle $L=\Lambda^{2}(M)$ of the canonical Spin ${ }^{c}$-structure.

\subsection{Seiberg-Witten Equations Without Self-Dua- lity on the $n$ - Manifolds}

Definition 3: Let $(M, g)$ be a $n$-dimensional $\operatorname{Spin}^{c}$ manifold. Then Seiberg-Witten Like equations for the $\operatorname{pair}(A, \Psi)$ is given by

$$
\begin{aligned}
& D_{A} \Psi=0 \text {, Dirac Equation } \\
& \rho^{+}\left(F_{A}\right)=\frac{1}{2}\left(\Psi \Psi^{*}\right)^{+}, \text {Curvature Equation }
\end{aligned}
$$

where $F_{A}$ is the curvature of $\mathrm{A}$ and $\left(\Psi \Psi^{*}\right)^{+}$is the orthogonal projection of $\Psi \Psi^{*}$ onto $W=\rho^{+}\left(\Omega^{2}(\mathrm{M}, \mathrm{i} \mathbb{R})\right)$. In the local orthonormal frame $\left\{e_{1}, \ldots, e_{n}\right\}$,

$$
\begin{aligned}
\left(\Psi \Psi^{*}\right)^{+} & =\operatorname{Proj}_{W}\left(\Psi \Psi^{*}\right) \\
& =\sum_{i<j} \frac{<\rho^{+}\left(e^{i} \wedge e^{j}\right), \Psi \Psi^{*}>}{<\rho^{+}\left(e^{i} \wedge e^{j}\right), \rho^{+}\left(e^{i} \wedge e^{j}\right)>} \rho^{+}\left(e^{i} \wedge e^{j}\right) .
\end{aligned}
$$

\section{Results and Discussion}

In this section, we write down the Seiberg-Witten-Like equation on 4 and 8 -dimensional manifolds. Then we compare the solution of these equations with the solution of classical Seiberg-Witten equations on $\mathbb{R}^{4}[8,9]$. Finally, we give a global solution to these equations on 8 -manifolds.

\subsection{Seiberg-Witten-like equation on $\mathbb{R}^{4}$}

In $M=\mathbb{R}^{4}$ case, the explicit form of the Dirac operator with respect to the $\operatorname{Spin}^{c}(4)-$ structure is given as follows:

$$
\begin{aligned}
\frac{\partial \psi_{1}}{\partial x_{1}} x_{4}+A_{1} \psi_{1}= & i\left(\frac{\partial \psi_{1}}{\partial x_{2}}+A_{2} \psi_{1}\right)+\frac{\partial \psi_{2}}{\partial x_{3}}+A_{3} \psi_{2} \\
& +i\left(\frac{\partial \psi_{2}}{\partial x_{4}}+A_{4} \psi_{2}\right) \\
\frac{\partial \psi_{2}}{\partial x_{1}}+A_{1} \psi_{2}= & -i\left(\frac{\partial \psi_{2}}{\partial x_{2}}+A_{2} \psi_{2}\right)-\frac{\partial \psi_{1}}{\partial x_{3}}-A_{3} \psi_{1} \\
+ & i\left(\frac{\partial \psi_{1}}{\partial x_{4}}+\frac{1}{2} A_{4} \psi_{1}\right) .
\end{aligned}
$$

The second equation of the Seiberg-Witten-like equations without self-duality is obtained as follows

$$
\begin{aligned}
& F_{12}+F_{34}=-\frac{i}{2}\left(\left|\psi_{1}\right|^{2}-\left|\psi_{2}\right|^{2}\right), \\
& F_{13}-F_{24}=\frac{1}{2}\left(\psi_{1} \overline{\psi_{2}}-\psi_{2} \overline{\psi_{1}}\right), \\
& F_{14}+F_{23}=-\frac{i}{2}\left(\psi_{1} \overline{\psi_{2}}+\psi_{2} \overline{\psi_{1}}\right) .
\end{aligned}
$$

Notice that, in the case of $M=\mathbb{R}^{4}$ Seiberg-Wittten-like equations without self-duality coincide with the classical Seiberg-Witen equations [8,9].

In the following we define the Seiberg-Witten-like equations on 8 -manifolds.

\subsection{Seiberg-Witten- like equation on $\mathbb{R}^{8}$}

By considering the following $\operatorname{Spin}^{c}-$ structure [5]:

$$
\kappa_{8}: \mathbb{R}^{8} \rightarrow \mathbb{C}^{16}
$$




$$
\kappa_{8}\left(\mathrm{e}_{\mathrm{i}}\right)=\left[\begin{array}{cc}
0 & \mu\left(\mathrm{e}_{\mathrm{i}}\right) \\
-\mu\left(\mathrm{e}_{\mathrm{i}}\right) & 0
\end{array}\right]
$$

where $e_{i}, i=1, \ldots, 8$ is the standart basis of $\mathbb{R}^{\mathbf{8}}, \mu\left(e_{1}\right)=$ $I_{d}$ is a $8 \times 8$ identity matrix , and for $i=1, \ldots, 8$ explicit form of $\mu\left(e_{i}\right)$ are given by

$$
\begin{array}{ll}
\mu(2)=I_{2} \otimes I_{2} \otimes m_{1}, & \mu(3)=I_{2} \otimes I_{2} \otimes m_{2}, \\
\mu(4)=i I_{2} \otimes m_{1} \otimes m_{1} m_{2}, & \mu(5)=i I_{2} \otimes m_{2} \otimes m_{1} m_{2}, \\
\mu(6)=-m_{1} \otimes m_{1} m_{2} \otimes m_{1} m_{2}, & \mu(7)=-m_{2} \otimes m_{1} m_{2} \otimes m_{1} m_{2}, \\
\mu(8)=-m_{1} m_{2} \otimes m_{1} m_{2} \otimes m_{1} m_{2} &
\end{array}
$$

where $I_{2}$ is a $2 \times 2$ identity matrix and

$$
m_{1}=\left[\begin{array}{cc}
i & 0 \\
0 & -i
\end{array}\right], \quad m_{2}=\left[\begin{array}{ll}
0 & i \\
i & 0
\end{array}\right] .
$$

By using (3.1) one can obtain explicit form of (2.4) and the solution of these equation can be obtained by the following pair,

$$
A=\sum_{i=1}^{8}-2 i x_{i} d x^{i}
$$

and

$$
\Psi=\left(0,0,0, e^{\sum_{j=1}^{8}-\frac{1}{2} x_{j}{ }^{2}}, 0,0, e^{\sum_{j=1}^{8}-\frac{1}{2} x_{j}{ }^{2}}, 0\right) .
$$

Here $(A, \Psi)$ is the local, non-trivial but flat $\left(i e . F_{A}=0\right)$ solution of the Seiberg-Witten-like equation without self-duality with respect to $M=\mathbb{R}^{8}$.

In the next subsection, a global solution to the Seiberg-Witten-like equations without self-duality is given on 8 -real-dimensional Kähler Manifolds.

\subsection{Seiberg-Witten-Like Equations on the 8 -Real-Dimensional Kähler Manifold}

Let $(M, g, J)$ be a 8 -real-dimensional Kähler manifold endowed with a canonical $\operatorname{Spin}^{c}-$ structure and $e_{1}, e_{2}=$ $J\left(e_{1}\right), e_{3}, e_{4}=J\left(e_{3}\right), e_{5}, e_{6}=J\left(e_{5}\right), e_{7}, e_{8}=J\left(e_{7}\right)$, be a local orthonormal frame with the dual basis $\left\{e_{1}, e_{2}, e_{3}\right.$, $\left.e_{4}, e_{5}, e_{6}, e_{7}, e_{8}\right\}$. Then the Kähler 2 -form has the form

$$
\Phi=e_{1} \wedge e_{2}+e_{3} \wedge e_{4}+e_{5} \wedge e_{6}+e_{7} \wedge e_{8} .
$$

Under the action $\Phi$, one gets the following decomposition

where

$$
\mathbb{S}=\mathbb{S}_{0} \oplus \mathbb{S}_{1} \oplus \mathbb{S}_{2} \oplus \mathbb{S}_{3} \oplus \mathbb{S}_{4},
$$

$$
\begin{aligned}
& \mathbb{S}_{0}=\{\Psi \in \mathbb{S} \mid \Phi \Psi=4 \mathrm{i} \Psi\}, \\
& \mathbb{S}_{1}=\{\Psi \in \mathbb{S} \mid \Phi \Psi=2 \mathrm{i} \Psi\}, \\
& \mathbb{S}_{2}=\{\Psi \in \mathbb{S} \mid \Phi \Psi=0\}, \\
& \mathbb{S}_{3}=\{\Psi \in \mathbb{S} \mid \Phi \Psi=-2 \mathrm{i} \Psi\}, \\
& \mathbb{S}_{4}=\{\Psi \in \mathbb{S} \mid \Phi \Psi=-4 \mathrm{i} \Psi\} .
\end{aligned}
$$

Accordingly, $f: i^{4} e_{1} \cdot e_{2} \cdot e_{3} \cdot e_{4} \cdot e_{5} \cdot e_{6} \cdot e_{7} \cdot e_{8}: \mathbb{S} \rightarrow$ $\mathbb{S}$ endomorphism, the complex spinor bundle $\mathbb{S}$ splits into

$$
\mathbb{S}=\mathbb{S}^{+} \oplus \mathbb{S}^{-}
$$

where

$$
\begin{aligned}
& \mathbb{S}^{+}=\mathbb{S}_{0} \oplus \mathbb{S}_{2} \oplus \mathbb{S}_{4} \cong \Lambda^{0,4}(M) \oplus \bigwedge^{0,2}(M) \oplus \Lambda^{0,0}(M), \\
& \mathbb{S}^{-}=\mathbb{S}_{0} \oplus \mathbb{S}_{3} \cong \Lambda^{0,3}(M) \oplus \Lambda^{0,1}(M) .
\end{aligned}
$$

Let $\Psi_{0}$ be a spinor in $\mathbb{S}_{4} \cong \Omega^{0,0}(M)$ corresponding to constant function 1 in the chosen coordinates

$\Psi_{0}=\left[\begin{array}{l}0 \\ 0 \\ 0 \\ 0 \\ 0 \\ 0 \\ 0 \\ 1\end{array}\right]$. By using $\Psi_{0}$, one has
$\frac{\left(\Psi_{0} \Psi_{0}{ }^{*}\right)^{+}}{2}=\left[\begin{array}{cccccccc}0 & 0 & 0 & 0 & 0 & 0 & 0 & 0 \\ 0 & 0 & 0 & 0 & 0 & 0 & 0 & 0 \\ 0 & \frac{-1}{4} & 0 & 0 & 0 & 0 & 0 & 0 \\ 0 & 0 & 0 & 0 & 0 & 0 & 0 & 0 \\ 0 & 0 & 0 & 0 & 0 & 0 & 0 & 0 \\ 0 & 0 & 0 & 0 & 0 & 0 & 0 & 0 \\ 0 & 0 & 0 & 0 & 0 & 0 & 0 & 0 \\ 0 & 0 & 0 & 0 & 0 & 0 & 0 & \frac{1}{4}\end{array}\right]$

Let $A_{0}$ be the connection on the $S^{1}$-principal bundle $P_{S^{1}}$ induced by means of the Levi-Civita connection $\nabla$ in the line bundle $L=\Omega^{0,2}(M)$ of the canonical $\operatorname{Spin}^{c}$ - structure [4]. Accordingly, the corresponding Dirac operator $D_{A_{0}}: \Gamma\left(\mathbb{S}^{+}\right) \rightarrow \Gamma\left(\mathbb{S}^{-}\right)$coincides with $\sqrt{2}\left(\overline{\partial_{0}} \oplus \overline{\partial_{2}{ }^{*}}\right)$. Also, the curvature of the connection 1 -form $A_{0}$ is given by

$$
F_{A_{0}}=i \rho_{\text {ric }}
$$

where $\rho(X, Y)=(X, Y)=g(X, J \circ \operatorname{Ric}(Y)) \quad$ and Ric: TM $\rightarrow$ TM denotes the Ricci tensor. Since the almost complex structure $\mathrm{J}$ and the Ricci tensor Ric commute, one has

$$
\begin{aligned}
\rho_{\text {ric }}= & -R_{11} e_{1} \wedge e_{2}-R_{33} e_{3} \wedge e_{4}-R_{13}\left(e_{1} \wedge e_{4}-e_{2} \wedge e_{3}\right) \\
& +R_{14}\left(e_{1} \wedge e_{3}-e_{2} \wedge e_{4}\right)-R_{15}\left(e_{1} \wedge e_{6}-e_{2} \wedge e_{5}\right) \\
& +R_{16}\left(e_{1} \wedge e_{5}+e_{2} \wedge e_{6}\right)-R_{17}\left(e_{1} \wedge e_{8}-e_{2} \wedge e_{7}\right) \\
& +R_{18}\left(e_{1} \wedge e_{7}+e_{2} \wedge e_{8}\right)-R_{35}\left(e_{3} \wedge e_{6}-e_{4} \wedge e_{5}\right) \\
& +R_{36}\left(e_{3} \wedge e_{5}+e_{4} \wedge e_{6}\right)-R_{37}\left(e_{3} \wedge e_{8}-e_{4} \wedge e_{7}\right) \\
& +R_{38}\left(e_{3} \wedge e_{7}+e_{4} \wedge e_{8}\right)-R_{55} e_{5} \wedge e_{6}-R_{77} e_{7} \wedge e_{8} \\
& -R_{57}\left(e_{5} \wedge e_{8}-e_{6} \wedge e_{7}\right)+R_{58}\left(e_{5} \wedge e_{7}-e_{6} \wedge e_{8}\right) .
\end{aligned}
$$

In the following a global solution is given for the appropriate Ricci tensor.

\section{Theorem 1.}

Let $(M, g, J)$ be an 8 -real-dimensional Kähler manifold. Then for a given negative and constant scalar curvature $s\left(A_{0}, \Psi=\sqrt{-2 s} \Psi_{0}\right)$ is the solution of the Seiberg-Witten-like equations without self-duality.

Proof. Since $\Psi=\sqrt{-2 s} \Psi_{0} \in \Omega^{0,0}(M)$ and $\Psi$ is the spinor field corresponding to the constant function 1 , by using (2.3), one gets $D_{A_{0}} \equiv 0$. Satisfying the curvature equation remains. To achive this, Ric must be taken as follows:

$$
\text { Ric }=\left[R_{i j}\right]_{8 \times 8}= \begin{cases}\frac{S}{8} & i=j \\ 0 & i \neq j,\end{cases}
$$


where $s$ is the negative and constant. By using Ric in (3.2), one gets $\rho^{+}\left(F_{A_{0}}\right)=i \rho^{+}\left(\rho_{\text {ric }}\right)$ which means $\rho^{+}\left(F_{A_{0}}\right)=\frac{\left(\Psi \Psi^{*}\right)^{+}}{2}$.

\section{Conclusion}

We give a global solution to the Seiberg-Witten-like equations on 8 -real-dimensional Kähler manifolds for a given negative and constant scalar curvature.

\section{Acknowledgement}

Author thanks referee for his/her valuable suggestion remarks regarding the manuscript.

\section{References}

1. Bilge, A.H, Dereli, T, Koçak, Ş, Monopole equations on 8-manifolds with Spin(7) holonomy, Communications in Mathematical Physics, 1999, 203(1), 21-30.

2. Değirmenci, N, Özdemir, N, Seiberg-Witten like equations on 8dimensionalmanifolds with structure group Spin(7), Journal of Dynamical System and Geometric Theories, 2009, 7(1), 21-39.

3. Donaldson, S.K, Seiberg-Witten equations and 4-manifold topology, Bulletin of the American Mathematical Society, 1996, 33, 45-70.

4. Friedrich, T, Dirac operators in Riemannian geometry; Grauate Studies in Mathematics 25, American Mathematical Society, 2000; pp 211.

5. Karapazar, Ş, Seiberg-Witten equations on 8-dimensional $S U(4)$-structure, International Journal of Geometric Methods in Modern Physics, 2013, 10(3), 1220032.

6. Morgan, J, Seiberg-Witten Equations and Applications to the topology of Smooth Manifolds; Princeton University Press, 1996; pp 130 .

7. Naber, G.L, Topology, geometry, and gauge fields; New York: Springer-Verlag, 1996; pp 437.

8. Salamon, D, Spin geometry and Seiberg-Witten invariants. Zürich: ETH, 1995; pp 599.

9. Witten, E, Monopoles and four manifolds, 1994, Mathematical Research Letters, 1994, 1, 769-796. 